\title{
Factores de riesgo asociados a los trastornos de la conducta alimentaria en estudiantes de ciencias de la Salud de la Universidad Andina del Cusco, 2019
}

\author{
Risk factors associateds with disorders of food behavior in student of Andina University of \\ Cusco, 2019
}

\author{
Karen A. Flores ${ }^{1,}$, K. Arenas ${ }^{1}$, L. Franco ${ }^{1}$, K. Huallpa ${ }^{1}$, B. Tacuri ${ }^{1}$, J. Pérez ${ }^{2}$ \\ ${ }^{1}$ Unive rsidad Andina del Cusco, Facultad de Ciencias de la Salud, Escuela de Medicina Humana Cusco, Perú. \\ 2 Universidad Andina del Cusco. Cusco, Perú \\ ”017200445i@uandina.edu.pe
}

\begin{abstract}
Resumen
Como antecedentes se menciona que los trastornos de conducta alimentaria representan la tercera enfermedad crónica más común entre las adolescentes llegando a una incidencia del 5\% según la OMS. El objetivo fue determinar los factores de riesgo asociados a los trastornos de la conducta alimentaria en estudiantes de la facultad de ciencias de la salud de la universidad Andina del Cusco, Perú. En cuanto al material y métodos empleados se realizó un estudio transversal analítico de diseño observacional. Se trabajó con una muestra de 264 estudiantes mayores de 16 años de la Facultad de Ciencias de Salud. Se empleó como instrumento el Eating attitudes test -26 y el test Body shape questionnaire. Los resultados permitieron determinar que 21 de ellos presenta 4 factores de riesgo: Imagen corporal con un 0R: 11.36, IC: [ 3.72-33.62] y p: 0.000005, Actitud alimentaria 0R: 40.65, IC: [ 11.63-174.9] y p: 0.0000, Autoestima con un 0R: 19.17, IC: [ 5.816-161.55] y p: 0.000021 y depresión con un 0R: 7.007, IC: [ 1.90-23.41] y p: 0.00169. La prevalencia del Trastorno de Conducta Alimentaria en los estudiantes es de $8 \%(n=21)$ de un total de 264. En conclusión, se llegó a demostrar que existe una asociación fuerte entre estos factores de riesgo y los Trastornos de la conducta alimentaria. Palabras clave: factores de riesgo, trastornos, conducta alimentaria, imagen corporal
\end{abstract}

\section{Abstract}

As background, it is mentioned that eating disorders represent the third most common chronic disease among adolescents, reaching an incidence of 5\% according to the WHO. The objective was to determine the risk factors associated with eating disorders in students of the Faculty of Health Sciences of the Andean University of Cusco, Peru. Regarding the material and methods used, an analytical cross-sectional study of observational design was carried out, including 264 students of the faculty of health sciences over 16 years. The Eating attitudes test - 26 and the Body shape questionnaire test were used as an instrument. Results: It was obtained that 21 of them present 4 risk factors: Body image with a 0R: 11.36, CI: [3.72-33.62] and p: 0.000005, Food attitude 0R: 40.65, CI: [11.63-174.9] and p: 0.0000, Self-esteem with a 0R: 19.17, CI: [5,816-161.55] and p: 0.000021 and depression with a 0R: 7.007, CI: [1.90-23.41] and p: 0.00169, the prevalence of Eating Disorder students is $8 \%(n=21)$ out of a total of 264. In conclusion, it was shown that there is a strong association between these risk factors and eating disorders.

Keywords: risk factors, disorders, eating behavior, body image

Citar como: Flores, KA., Arenas, K., Franco, L., Hallpa, K., Taxuri, B., Pérez, J. (2019). Factores de riesgo asociados a los trastornos de la conducta alimentaria en estudiantes de ciencias de la Salud de la Universidad Andina del Cusco, 2019. Rev Yachay, 8(1),550-552.

Recibido: 30-07-2019; Aceptado: 07-10-2019; Publicado: 12-12-2019

\section{Introducción}

Los trastornos en la conducta alimentaria (TCA) son una agrupación de enfermedades que se caracterizan por la aparición de cambios en la forma de comer por exceso o por defecto (Gómez, 2009), lo que conlleva a problemas físicos; además de afectar al funcionamiento psicológico y social del individuo y su entorno (Fundación Imagen y Autoestima, 2013). Se trata de trastornos mentales asociados a 
factores de riesgo: insatisfacción corporal, depresión, autoestima, actitud alimentaria, la imagen corporal y lo que esta representa (Anorexia y Bulimia) (American Psychiatric Association, 2014).

En la actualidad que la mayoría de adolescentes y jóvenes son los que presentan este tipo de trastornos alimentarios (Gómez, García \& Corral, 2009). Entonces, este trastorno aparece con mayor frecuencia en muchachas adolescentes y mujeres jóvenes (Chinchilla, 2003). Las consecuencias de padecer estos trastornos de conducta alimentaria debido a los factores de riesgo son la presencia de distintos síntomas, tanto como la disminución anormal de la presión arterial, debilidad muscular, deshidratación severa, sensación de desmayo, fatiga, piel seca, pérdida de cabello, desbalance electrolítico y entre otros [Torres (2019), Chávez \& Lumley (2019)].

De acuerdo a un estudio en la ciudad de Cusco titulada "Evaluación de la predisposición a trastornos de conducta alimentaria en adolescentes del Cusco 2012", valora la predisposición a Trastornos de Conducta Alimentaria en relación a las actitudes alimentarias anómalas, distorsión e insatisfacción de la imagen corporal, en los adolescentes de la ciudad del Cusco. Con una población de 126 se obtuvo como resultado, que el 15\% del total de adolescentes presentó predisposición a TCA, de los cuales el 10.3\% correspondió al sexo femenino y $4.7 \%$ al masculino. El $16 \%$ presentó morbilidad frente a las actitudes alimentarias anómalas, el $4 \%$ presentó morbilidad aguda, 73\% insatisfacción de la imagen corporal y el 77\% estado nutricional normal (Quispe, 2012).

Sin embargo, estas referencias bibliográficas subestiman que el Perú se encuentra dentro de un modelo de transición epidemiológica, en la cual la población se está viendo influenciada por diferentes medios de comunicación, instituciones sociales y de cultura, estas nuevas formas de vida que se andan generando están condicionando la patología médica y nutricional de los habitantes, especialmente en jóvenes. Por ello, el siguiente estudio tiene como objetivo general identificar los factores de riesgo asociados a los trastornos de la conducta alimentaria en estudiantes de la Facultad de Ciencias de la salud de la Universidad Andina del Cusco 2019.

\section{Materiales y métodos}

El diseño de estudio empleado en este trabajo corresponde al tipo observacional transversal, realizado en los estudiantes de la Facultad de Ciencias de la Salud de la Universidad Andina del Cusco 2019.

La población estuvo constituida por los estudiantes de la Facultad de Ciencias de la Salud de la Universidad Andina del Cusco, para la muestra se seleccionó de manera aleatoria 264 estudiantes.

\section{Variables de estudio}

a) Factores de riesgo

Los factores de riesgo evaluados fueron cuatro: Actitud alimentaria, imagen corporal, autoestima y depresión con llevan importantes alteraciones conductuales, como consecuencia del intento por controlar el cuerpo y el peso.

b) Trastornos de la conducta alimentaria

Los trastornos de la conducta alimentaria evaluados fueron: Anorexia, bulimia y otros que se caracterizan por la aparición de cambios en la forma de comer por exceso o por defecto, lo cual con lleva a conflictos psicológicos no resueltos.

c) Otras variables

Se consideraron: Sexo, edad y semestre académico.

\section{Aspectos éticos}

El proyecto de investigación se basó en el respeto establecido en el Código de Ética y Deontología del Colegio Médico del Perú (2007) y a la Declaración de Helsinki. Busca respetar los principios bioéticos de los seres humanos, priorizando la protección de los individuos en una investigación que respete su dignidad, salud y derechos. Antes del desarrollo de la encuesta se explicó el correcto llenado de la encuesta. Se obtuvo el consentimiento informado de cada participante. Los datos recolectados durante el estudio se utilizaron solo para fines de investigación y se manejaron como confidenciales

\section{Procedimientos o intervenciones}

La recolección de datos se dio por medio de instrumentos para la recolección de datos; test Body Shape Questionnaire, EAT26 y, por último, datos generales de la población.

\section{Análisis de datos}

Los datos recogidos se registraron y clasificaron en Microsoft office y Excel 2013 Posteriormente, se hizo el análisis en Epi Info 7.2.2.6 y Test Exact Fisher. Se presentaron los resultados de las variables en frecuencias y porcentajes. La fuerza de asociación se describió a través del (OR) e (IC95\%). Se consideró un p<0,05.

\section{Resultados}

Se contactó con 264 adolescentes, de los cuales el $71 \%$ de encuestados fue de género femenino. 21 estudiantes presentaron factores de riesgo asociados a los trastornos de conducta alimentaria en la Universidad Andina del Cusco 2019.

En cuanto a las Escuelas Profesionales encuestadas, el 34\% Medicina Humana; el 33\% Obstetricia, 14\% Psicología, 14\%, Enfermería y $5 \%$ Estomatología. 
Tabla 1

Factores de riesgo y Trastornos de la conducta alimentaria en adolescentes $18 \pm 1.5$ años

\begin{tabular}{lccccc}
\hline & \multicolumn{1}{c}{ Varones } & \multicolumn{2}{c}{ Mujeres } & \multicolumn{1}{c}{$\boldsymbol{P}$} \\
\hline & $\mathrm{N}=76 \%$ & $\mathrm{~N}=188 \%$ & \\
\hline Depresión & 2 & $3 \%$ & 4 & $2 \%$ & 0.00169 \\
\hline Autoestima & 4 & $5 \%$ & 6 & $3 \%$ & 0.000021 \\
\hline Imagen corporal & 3 & $4 \%$ & 7 & $4 \%$ & 0.00005 \\
\hline Actitud alimentaria & 6 & $8 \%$ & 11 & $6 \%$ & 0.0000
\end{tabular}

Tabla 2

Factores de riesgo y Trastornos de la conducta alimentaria en adolescentes $18 \pm 1.5$ años de acuerdo a la escuela profesional

\begin{tabular}{lcc}
\hline & Cantidad & Con TCA \\
\hline Enfermería & 3 & $14 \%$ \\
\hline Estomatología & 1 & $5 \%$ \\
\hline Medicina Humana & 7 & $33 \%$ \\
\hline Psicología & 3 & $14 \%$ \\
\hline Obstetricia & 7 & $33 \%$ \\
\hline Tecnología médica & 0 & 0 \\
\hline
\end{tabular}

\section{Conclusión}

El presente estudio tiene como muestra un total de 264 estudiantes de la Facultad de Ciencias de la salud de la Universidad Andina del Cusco, de los cuales el $71 \%(n=188)$ fueron de sexo femenino y un $29 \%(n=76)$ de sexo masculino a quienes se les realizó un cuestionario para determinar si presentan o no trastorno de conducta alimentaria y los factores asociados a este concluyendo lo siguiente:

- La prevalencia del Trastorno de Conducta Alimentaria en estudiantes de la Facultad de ciencias de la Salud de la Universidad Andina del Cusco es de $8 \%(n=21)$ de un total de 264 estudiantes encuestados.

- Se evidenció una relación estadísticamente significativa entre la presencia de Trastorno de Conducta Alimentaria y los factores de riesgo que se estudiaron, imagen corporal con un OR: 11.36, actitud alimentaria OR: 40.65, autoestima OR: 19.17, depresión OR: 7,077 .

- Del mismo modo, se evaluó la presencia de depresión y trastorno de conducta alimentaria donde se obtuvo que un 32\%(n=6) presentó ambos trastornos y $68 \%$ solo depresión.

\section{Referencias bibliográficas}

Gomez J, Garcia M, Corral L.(2009) Convivir con Trastornos de la Conducta Alimentaria: Anorexia, Bulimia y Trastorno por Chinchilla A. (2003) Trastornos de la conducta atracones; $1^{\circ}$ ed. Buenos Aires; Madrid: Medica Panamericana. Pág. 3,10-12.

Fundación imagen y autoestima. Trastornos de la conducta alimentaria. [internet]. México; 2013. [Consultado 8 de marzo de 2019]. Disponible en : http://www.fima.org/es/trastornos-relacionados

American Psychiatric Association.(2014) Manual diagnóstico y estadístico de los causas y características. [Internet]. trastornos mentales: DSM-5; $5^{\circ}$ ed. Chavez M, Lumley D.(2017) Bulimia nerviosa. Madrid: MédicaPanamericana. alimentaria: Anorexia y bulimia nerviosas, obesidad y atracones; $1^{\circ}$ ed.Barcelona; España: MASSON S.A.; Capitulo 1

Torres A. (2019) Tipos de anorexia, síntomas, Psicología y Mente. [Consultado 14 de abril del 2019]. Disponible en: https://psicologiaymente.com/clinica/tip os-anorexia NIMH . [Internet]. [consultado 14 de Mar 2019]. Disponible en: https://espanol.womenshealth.gov/menta 1-health/mental-health-conditions/eatingdisorders/bulimia-nervosa

Quispe K. (2012) Evaluación de la predisposición a trastornos de conducta alimentaria en adolescentes del Cusco 2012. Repositorio Unsaac [Tesis Internet]. [Consultado el 19 de marzo de 2019]; Disponible en: http://especializada.unsaac.edu.pe/cgibin/koha/opacdetail.pl?biblionumber $=15021$ 